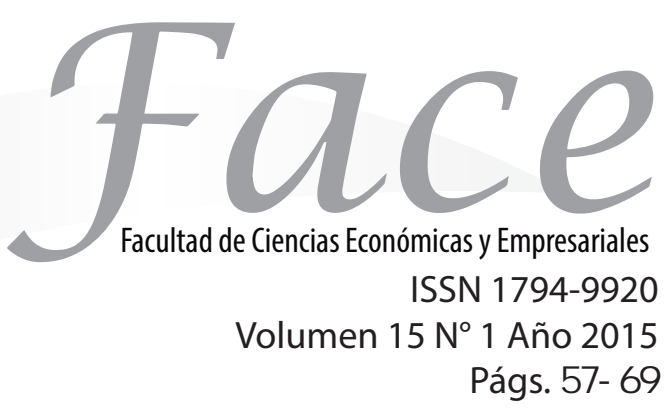

\title{
DIAGNÓSTICO DEL CLIMA LABORAL Y ORGANIZACIONAL DE LOS CONDUCTORES DE LOS CAMPACTADORES DE LA EMPRESA DE ASEO DE MEDELLÍN- EMVARIAS
}

\author{
Mario Javier Naranjo Otalvaro*
}

Fecha de Recepción: 27 de Febrero 2015

Fecha de Aprobación: 29 de Junio 2015

\section{Resumen:}

El artículo muestra los resultados de la investigación desarrollada en la empresa de aseo de la ciudad de Medellín -Emvarias- para conocer las necesidades de formación y bienestar de los conductores, reflejadas en el clima laboral en el que se desempeñan. Se identificaron fortalezas y aspectos por mejorar, propendiendo porque el componente humano y social al interior de la empresa se potencialicen, de forma que el crecimiento de los capitales físico, económico y humano sea integral. La investigación fue de tipo descriptiva bajo técnicas de corte cualitativo y cuantitativo.

Palabras Claves: Ambiente laboral, Clima organizacional, Talento humano, Empresa, Necesidades, Dimensiones. 


\title{
DIAGNOSIS OF THE WORK AND ORGANIZATIONAL ENVIRONMENT OF CAMPACTOR DRIVERS OF THE SANITATION COMPANY OF MEDELLIN - EMVARIAS.
}

\begin{abstract}
:
This article shows the results of the research carried out in the sanitation company -Emvarias, Medellin so as to understand the driver's welfare and training needs, reflected in their work environment. The study identified a number of strengths and areasthat can be improved in order to enhance the company's human and social components, so that the human, economic and physical capitals are integral. A descriptive research method was used combined with quantitative and qualitative techniques.
\end{abstract}

keywords: Work environment, Organizational climate, Human talent, Enterprise, Needs, Dimensions.

\section{DIAGNÓSTICO DO AMBIENTE DE TRABALHO E CLIMA ORGANIZACIONAL DOS CONDUTORES DE COMPACTADORES DA EMPRESA DE SANEAMENTO DE MEDELLÍN- EMVARIAS}

\begin{abstract}
Resumo:
O artigo mostra os resultados da pesquisa realizada na empresa de saneamento da cidade de Medellín Emvarias - com o objetivo de entender as necessidades de formação e bem-estar dos condutores, refletido no ambiente de trabalho no qual se desempenham. Identificaram-se pontos fortes e áreas de melhoria, com a finalidade de potenciar o capital humano e social da empresa, de forma que o crescimento dos capitais físico, econômico e humano seja integral. A pesquisa foi descritiva e se utilizou técnicas qualitativas e quantitativas.
\end{abstract}

Palavras-Chave: Ambiente de Trabalho, Clima Organizacional, Talento Humano, Empresas, Necessidades, Dimensões. 
Mario Javier Naranjo Otalvaro.

\section{Introducción:}

Se conoce como clima laboral al medio ambiente humano y físico, en el que se desarrolla el trabajo cotidiano; este es esencial para la satisfacción del empleado, el cual determina la calidad y el aumento de la productividad; está relacionado con el "saber hacer" del trabajador, con los comportamientos de las personas, con su manera de trabajar y de relacionarse, con su interacción con la empresa, con las máquinas que se utilizan y con la propia actividad de cada uno.

Existen dos tipos de necesidades generales en la vida de todo ser humano: materiales y de espíritu. Para satisfacer las necesidades materiales, es necesario tener comida, ropa, vivir en un lugar adecuado, tener dinero para sufragar la educación de los hijos y cubrir unos niveles mínimos de bienestar. Sin embargo las necesidades de espíritu muchas veces no están cubiertas en el ser humano: ser dueños de nuestro propio destino, encontrar aliciente en el trabajo y en la vida personal (amigos, familia, amor). La sociedad actual se caracteriza por enfatizar y perseguir las necesidades materiales a cambio de las necesidades de espíritu.

Actualmente la productividad de los trabajadores, además del clima laboral en el que estén inmersos, está ligada a las compensaciones e incentivos que ofrece la organización, lo cual genera espacios de crecimiento en todos los aspectos $y, a$ su vez, redunda en mejores resultados para la organización.

Este aspecto debe estar enmarcado en el plan de bienestar laboral que cada empresa contempla desde el área de talento humano, donde se establecen puntos clave para contribuir con jornadas recreativas, deportivas y culturales que no generan mayor costo, pero sí estimulan en el empleado sentido de pertenencia y gusto por su tarea diaria (Ceballos, 2014).

Una de las grandes preocupaciones de las compañías tiene que ver con la capacidad de ofrecer valores agregados a sus colaboradores Vecino (2013); además el bienestar laboral está orientado a mejorar el clima de las empresas y mantener la energía en los equipos de trabajo; en ese orden de ideas, es necesario involucrar a la alta gerencia, comunicar a todos los niveles de la organización y además, informar de manera externa con el fin de incrementar la reputación e imagen como una de las mejores empresas para trabajar. De otro lado, las compensaciones deben incluir el entorno inmediato del empleado como una vía efectiva para mantener un estado emocional estable, porque "no tiene sentido un programa que solo apoye al colaborador sin tener en cuenta a su familia". (Ceballos, 2014). Con las premisas anteriores, se adelantó una investigación en la empresa de aseo de la ciudad de Medellín E.S.P. EMVARIAS; la cual consistió en un diagnóstico para conocer el clima laboral y organizacional de los conductores que operan los compactadores encargados de la recolección, separación y disposición final de los residuos sólidos urbanos de la ciudad.

Los resultados del mismo permiten un mayor conocimiento de las condiciones reales en que laboran actualmente los conductores del parque automotor de Emvarias; una vez conocida esta situación y asumida en su integralidad por la alta gerencia de la compañía, se espera surta un proceso de mejoramiento cualitativo y cuantitativo en las distintas áreas que la conforman.

\section{Metodología:}

La metodología utilizada se basó en el estudio de encuesta tipo descriptivo de corte cualitativo y cuantitativo, el mismo que posibilitó dividir el proceso de investigación en cuatro fases, a saber:

Planteamiento de objetivos y preparación de instrumento de recogida de información. Planificación del muestreo: la población finita seleccionada estuvo conformada por 224 conductores, de los cuales 184 lograron responder la encuesta, correspondiente al $82 \%$ del universo.

Recogida de datos: se dispuso la población sujeto de investigación en tres jornadas mañana, tarde y noche en tres días distintos. Análisis e interpretación de los datos: se hizo un análisis descriptivo cuantitativa y numéricamente a partir del instrumento diligenciado por parte de la población encuestada.La muestra seleccionada correspondió al universo de la población conformada por 224 conductores, de los cuales 184 lograron responder la encuesta, correspondiente al $82 \%$ del universo. 


\section{Resultados y discusión:}

Los resultados se presentan condensados en 8 bloques temáticos (dimensiones), que facilitan el conocimiento y la interpretación de los aspectos básicos necesarios para lograr el objetivo del estudio. Dichas dimisiones son:

\subsection{Dimensión socio-demográfica}

Son el conjunto de características biológicas y socioeconómico culturales que están presentes en la población sujeta a estudio, tomando aquellas que puedan ser medibles (Rubines, s.f.). Esta categoría de análisis permite conocer las características personales, familiares, económicas y sociales de la población sujeto de estudio.

Durante la aplicación del instrumento los conductores trabajaban porcentualmente con los siguientes contratistas (empresas particulares que tienen contratadoOutsourcing-del servicio de recolección de residuos orgánicos urbanos, con la entidad del Estado Emvarias); en orden descendente según lo muestra la gráfica.

Tabla No 1

\section{Empleador}

Frecuencia

\begin{tabular}{lcc} 
Funtraev & 48 & 26,1 \\
\hline Poliservicios & 30 & 16,3 \\
\hline Corprudec & 26 & 14,1 \\
\hline Cludeco & 22 & 12.0 \\
\hline $\begin{array}{l}\text { Recuperación } \\
\text { paz }\end{array}$ & 18 & 9,8 \\
\hline Corjunipaz & 16 & 8,7 \\
\hline Corpuem & 13 & 7,1 \\
\hline Cortrinidad & 11 & 6.0 \\
\hline Total & $\mathbf{1 8 4}$ & $\mathbf{1 0 0 , 0}$ \\
\hline
\end{tabular}

Fuente: propia
En cuanto al lugar de residencia, el $66.6 \%$ vive en Medellín; el resto en orden porcentual se distribuye en otros municipios del área metropolitana de departamento de Antioquia (Colombia) a saber: $21 \%$ en Bello; $5 \%$ en Itagüí; $3 \%$ en Copacabana; y los demás en Caldas, Barbosa, Envigado y La estrella; dado que los turnos establecidos por las empresas contratistas que prestan el servicio de aseo de la ciudad son tres por día, esta información puede facilitar una mayor organización de los mismos de acuerdo al lugar en que residen, para facilitarles el desplazamiento desde su lugar de residencia al lugar donde se encuentra el compactador (plaza de ferias).

\section{Gráfica No 1: Edad}

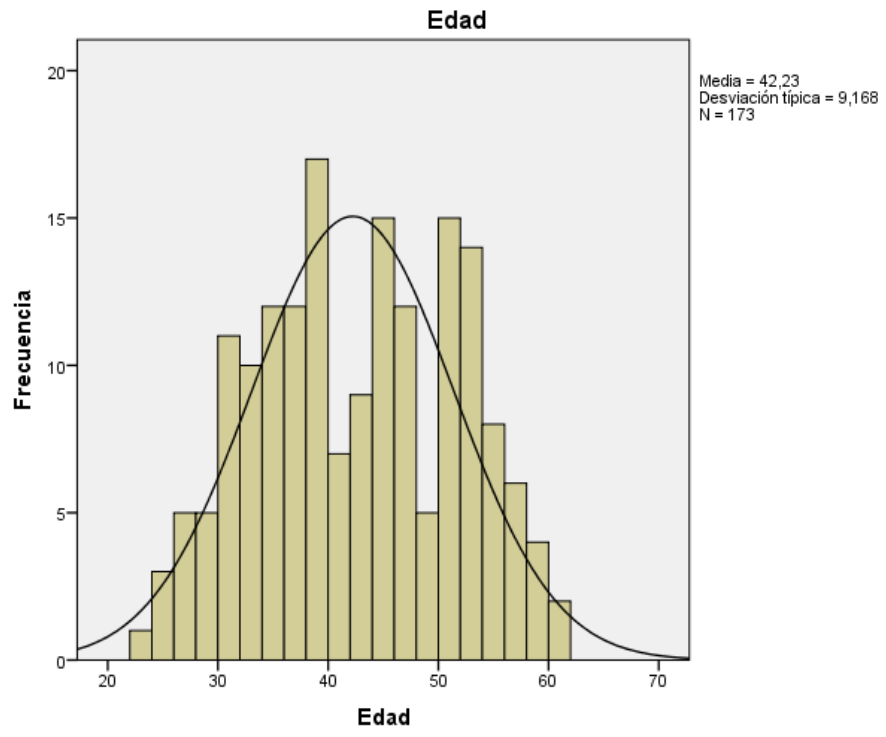

La edad promedio es de 42 años; representa un grupo muy heterogéneo en edades y con un potencial laboral importante, que podría ser aprovechado por el contratista con el que presten sus servicios, para garantizar una mayor productividad, dado que es la edad en la que se espera el ser humano tenga aspectos claves de su proyecto de vida muy claros, entre ellos el tener un oficio o una profesión ya muy consolidada, que para el caso, el promedio es de 21 años de experiencia como conductores, convirtiéndose este en un factor importante que permite pensar que la operación de los vehículos compactadores está en manos de expertos, lo que debe garantizar seguridad $y$ 


\section{DIAGNÓSTICO DEL CLIMA LABORAL Y ORGANIZACIONAL DE LOS CONDUCTORES DE LOS CAMPACTADORES DE LA EMPRESA DE ASEO DE MEDELLÍN- EMVARIAS}

Mario Javier Naranjo Otalvaro.

confianza no sólo a la empresa contratista, sino también a la ciudadanía.

Tabla No 2: Estado Civil

\begin{tabular}{lcc}
\hline \multicolumn{3}{c}{ Estado civil } \\
\hline & Frecuencia & $\begin{array}{c}\text { Porcentaje } \\
\text { válido }\end{array}$ \\
\hline Soltero & 10 & 5,4 \\
\hline Casado & 94 & 51,1 \\
\hline Unión libre & 71 & 38,6 \\
\hline Separado & 7 & 3,8 \\
\hline Viudo & 2 & 1,1 \\
\hline Total & 184 & 100,0 \\
\hline
\end{tabular}

Fuente: propia

También es de esperar que para la etapa de vida en la que se encuentra el promedio de los conductores, ya hayan consolidado su propio grupo familiar; que para el caso son casados el 51,1\% y el $38.6 \%$ viven en unión libre; el promedio de hijos del total de la población es de 2.1; es necesario indicar que el $35.8 \%$; tienen familias conformadas por dos hijos; seguidos por familias por un solo hijo $24.4 \%$; las familias más numerosas están conformadas hasta por cinco hijos 1.7\%; y no tienen hijos el 5.1\%; el número promedio de hijos por familias corresponde a la media del crecimiento demográfico de la población colombiana de los últimos años según el DANE. De todos ellos, el $83.1 \%$ vive con la esposa, y el $73.2 \%$ con sus hijos; indica lo anterior que la mayoría conforman hogares nucleares, fundamentales para la cohesión familiar y social. El $3.3 \%$ viven solos; tendencia muy marcada en estos tiempos de crisis de la institución familiar.

De los que tienen hijos, El $46.5 \%$ adelantan estudios de bachillerato, y el $40.7 \%$ estudios primarios; lo que indica que son familias en su mayoría muy jóvenes dedicadas a la formación de niños y adolescentes; adelantan estudios universitarios el $7.6 \%$ y trabajan el $30.2 \%$.
Tabla No 3: Estrato

\begin{tabular}{|c|c|c|}
\hline \multicolumn{3}{|c|}{ Estrato } \\
\hline & Frecuencia & $\begin{array}{c}\text { Porcentaje } \\
\text { válido }\end{array}$ \\
\hline 1 & 26 & 14,3 \\
\hline 2 & 93 & 51,1 \\
\hline 3 & 57 & 31,3 \\
\hline 4 & 4 & 2,2 \\
\hline 5 & 1 &, 5 \\
\hline 6 & 1 &, 5 \\
\hline Total & 182 & 100,0 \\
\hline $\begin{array}{l}\text { Perdidos } \\
\text { Sistema }\end{array}$ & 2 & \\
\hline Total & 184 & \\
\hline
\end{tabular}

Fuente: propia

El $65.4 \%$ pertenecen a los estrato socioeconómico 1 y 2, lo que según indicadores económicos oficiales se clasifican como familias de bajos recursos económicos; el 3.2\% de los conductores pertenecen a los estratos 4, 5 y 6, considerados según esos mismos indicadores, como familias de clase media y alta; se destaca así mismo que el $31.3 \%$ pertenecen al estrato 3 ; de éstos el $51.6 \%$ viven en casa arrendada y el $33.7 \%$ son propietarios de la vivienda que habitan, resultado acorde con los niveles de ingreso de los encuestados, que para el caso son entre uno y dos smlv; para el $52.5 \%$ de los conductores; y para el $1.7 \%$ es mayor a cuatro smlv; donde la adquisición de vivienda propia se dificulta, entre otras cosas porque la capacidad de ahorro es mínima o se carece de dicha cultura. Respecto al nivel académico, el $49.2 \%$ tienen bachillerato completo; y un $2.2 \%$ tienen estudios técnicos y/o adelantan estudios universitarios; el resto tienen bachillerato incompleto, primaria completa e incompleta. 
Tabla No 4: Nivel Académico

\begin{tabular}{lcc}
\hline & Nivel académico \\
\hline & Frecuencia & $\begin{array}{c}\text { Porcentaje } \\
\text { válido }\end{array}$ \\
\hline Primaria incompleta & 12 & 6,6 \\
\hline Primaria completa & 15 & 8,2 \\
\hline $\begin{array}{l}\text { Bachillerato } \\
\text { incompleto }\end{array}$ & 53 & 29,0 \\
\hline Bachillerato completo & 90 & 49,2 \\
\hline Estudios técnicos & 9 & 4,9 \\
\hline Estudios tecnológicos & 2 & 1,1 \\
\hline $\begin{array}{l}\text { Estudios } \\
\text { universitarios }\end{array}$ & 2 & 1,1 \\
\hline Total & 183 & 100,0 \\
\hline Perdidos Sistema & 1 & \\
\hline Total & 184 & \\
\hline
\end{tabular}

Fuente: Propia

Los resultados en el nivel de formación académica permiten resaltar que casi la mitad de los conductores está habilitado para continuar sus estudios superiores; los que no lo están, es deseable que la empresa formalizara convenios interinstitucionales con instituciones educativas, para que puedan terminar su bachillerato a través de la figura de la validación, y de esta manera poderse cualificar profesionalmente en los niveles técnico, tecnológico, ingenieril, e incluso a nivel de postgrado, en instituciones universitarias que ofrezcan estos ciclos de formación.

A pesar de ser una población con niveles de escolaridad medios o bajos, es una gran oportunidad que tiene la empresa de propiciarles a los conductores y su núcleo familiar posibilidades de acercamiento con las Instituciones Universitarias del Área Metropolitana de departamento de Antioquia, tanto para ellos como para sus hijos, con el fin de que conozcan la oferta académica de programas técnicos, tecnológicos, profesionales y de postgrado; en las que se puedan formar, en áreas relacionadas con su saber hacer, como lo son la mecánica automotriz, industrial, eléctrica, electrónica, mecatrónica, bioelectrónica, producción industrial; entre otras; lo anterior les podría garantizar mejores opciones y condiciones de vida tanto a nivel personal como familiar; consolidando proyectos de vida mucho más concretos.

\subsection{Motivación satisfacción}

García(2011) refiere que la motivación es una variable que influye en mayor medida que la satisfacción, en el desempeño y resultados productivos; la satisfacción y la motivación no son dependientes entre ellas; la motivación suele plantearse en forma de expectativas y de experiencias o de contribuciones y retribuciones mediadas por las posibilidades de cambio o de transformación que puedan sufrir; las expectativas expresan el conjunto de valores que el individuo ha ido asimilando y que quiere ver realizados en su ámbito del trabajo; así, la satisfacción será el resultado de lo que el individuo espera obtener y lo que obtiene realmente. Esta categoría permite identificar aspectos clave del conductor, relacionados con el trabajo que realiza y la posibilidad del desarrollo personal; así mismo, permite evaluar el equilibrio que hay entre el trabajo y las diferentes esferas que atañen a la vida del trabajador, tales como la familia, la gestión del tiempo libre, el desempeño laboral y la salud, tanto física como emocional.

Esta categoría permite reafirmar que para toda organización es muy importante que el talento humano, razón de ser de cualquier institución, mantenga expectativas positivas frente a la vida, que sean elementos motivadores expresados a través del conjunto de valores que todo ser humano asimila y practica a lo largo de su existencia, y que aspira ver realizadas en todos los ámbitos de desempeño, especialmente los más relevantes: el familiar y el laboral o profesional; la investigación mostró que el $\mathbf{9 0 . 8 \%}$ de los conductores manifestó estar muy satisfecho con su vida familiar; respecto a qué tan satisfechos se sienten con el ambiente de trabajo, el 56\% respondió sentirse muy satisfecho; aunque es un porcentaje importante, lo deseable para cualquier organización es que fuera del 100\%, porque un ambiente de trabajo acogedor, ameno, que haga sentir satisfecho al trabajador, no solamente es clave para la productividad de la empresa, sino que propicia un mayor sentido de pertenencia hacia la misma; generando un mejor compañerismo y respeto hacia ellos, sus compañeros, y favoreciendo todo el entorno que los rodea de manera muy positiva. 
Mario Javier Naranjo Otalvaro.

El resultado anterior, puede tener una relación directa con el $95.7 \%$ de los conductores que manifiesta no recibir beneficios adicionales al salario y las prestaciones sociales de parte del contratista o empleador; es bien sabido que cuando el trabajador goza de algunos beneficios adicionales a los meramente legales, no solamente se van a sentir más motivados, sino más agradecidos; detalles pequeños pero muy significativos para cualquier trabajador como programarles integraciones en fechas especiales como el día de trabajador, amor y amistad, navidad, una nota de felicitación con motivo de su cumpleaños, el nacimiento de un hijo, etc.; así como posibilitarles la adquisición de vivienda a través de algún tipo de préstamo, darles los útiles escolares a los hijos que adelanten la formación básica, genera una relación de gana-gana entre trabajador y empresa altamente beneficiosa.

Un pago justo es la condición más importante para cualquier trabajador, y el generador de una de las más importantes motivaciones; el $84.2 \%$ considera que no le pagan lo justo por su trabajo; resultado que debe ser motivo de una reflexión cuidadosa por parte de los contratistas o empleadores para evitar hacia el futuro dificultades en el ambiente laboral que puedan ser perturbadores para la buena marcha de la empresa y del cumplimiento de su misión institucional.

No obstante el resultado anterior, es muy significativo que el $95.7 \%$ de los conductores afirme que les gusta mucho el trabajo que realizan; siendo esta una gran oportunidad para que los directivos los empoderen más de su rol para hacerlos más productivos; los contratistas actuales o los que en el futuro vayan a operar el sistema de recolección de residuos sólidos en la ciudad, deberán definir unas políticas de bienestar laboral que les garantice una seguridad mínima en la continuidad de los mismos, que preferiblemente debiera ser a término indefinido; ya que a pesar de que un $64.7 \%$ manifestó sentir seguridad por conservar su empleo, un $21.7 \%$ afirma no tenerla; lo anterior se puede entender por la diversidad de operadores que prestan el servicio de recolección de basuras en la ciudad, pues indica que no todos les brindan las mismas condiciones laborales a los conductores que laboran con ellos; también tiene una asociación directa con el desarrollo de su proyecto de vida, que para el $62.5 \%$ de los encuestados el empleo que actualmente tienen, sí contribuye a su desarrollo; frente al $7.6 \%$ que respondió no contribuirle; resultado que puede ser aprovechado por parte de los empleadores para desarrollar actividades de empoderamiento y sentido de pertenencia hacia la empresa que posibilite involucrar el entorno familiar de los conductores, habida cuenta que el $80.9 \%$ su tiempo libre prefiere pasarlo con su familia, el $42.6 \%$ lo dedica a las labores del hogar, y el resto a descansar, practicar algún deporte y a estudiar; guarda una estrecha relación este resultado con respecto al porcentaje de casados que son la mayoría y que conviven con sus esposas e hijos, que conforman hogares nucleares y funcionales.

La salud de los encuestados por las cargas y exigencias laborales se ve comprometida para el $48.9 \%$, frente al $12.5 \%$ que responde verse muy a menudo comprometida; esta respuesta guarda relación con el promedio de edad y experiencia en el oficio que es de 42 y 20 años respectivamente; es normal que bajo esas condiciones, los problemas de salud empiecen a aflorar, lo que debe permitirles por parte de los empleadores desarrollar políticas de salud ocupacional para prevenir y atender oportunamente episodios de morbilidad que puedan convertirse en enfermedades profesionales, con niveles de incapacidad poco deseables para cualquier empresa, y la baja en la productividad que esta trae consigo; es necesario entonces que los problemas de estrés, irritabilidad, ansiedad que al $46.7 \%$ de los conductores le está generando el trabajo que realizan en algunas ocasiones, y al $8.7 \%$ que muy a menudo se les presenta, sean cobijados por estas políticas para evitar consecuencias mucho más graves. Los problemas de salud más significativos que han tenido los conductores por causas laborales son: estrés, problemas respiratorios, dolor de espalda crónico, problemas en los ojos, diabetes, cansancio físico crónico, problemas de columna, hernia discal, problemas de sueño por turnos, dolores lumbares, entre otros.

Otro factor diferenciador para estimular la motivación en el trabajo, es que los trabajadores puedan contar con políticas administrativas por parte de los empleadores que les permita contar con los permisos que todo trabajador regularmente 
necesita para atender compromisos personales 0 familiares que sólo él puede atender; el $52.7 \%$ de

los encuestados afirma que siempre les dan permiso para atender este tipo de compromisos; frente al $16.8 \%$ que respondió nunca o casi nunca. Lo anterior también se puede entender porque el contratista no es el mismo para todos los conductores, circunstancia nada conveniente porque es susceptible de generar malestar laboral entre el grupo de conductores; siendo algo a lo que todos deben tener derecho.

Los problemas económicos son generadores de dificultades en el desempeño laboral para el $50 \%$ de los encuestados que respondió en algunas ocasiones verse afectado; y el $8.7 \%$ respondió sentirse muy afectado; esta relación es muy común en muchos de los trabajadores; los empleadores para atenuar un poco esta realidad, y garantizar una mayor motivación con su trabajo, deberían ponderar más al ser humano y todas las complejidades que este maneja y menos al trabajador; si esto lo contemplan dentro de sus planes estratégicos las oficinas de talento humano, encargadas del bienestar laboral del trabajador, el resultado muy seguramente será rescatar la resiliencia que todas las personas son capaces de desarrollar; sobre todo si se tiene en cuenta que para el $80.9 \%$ de los encuestados las dificultades familiares no afectan su desempeño laboral.

Para el $51.6 \%$ de los encuestados, la principal necesidad o preocupación manifestada es por la educación de sus hijos; para el $46.8 \%$ por la estabilidad laboral; el $33.2 \%$ por las necesidades básicas; y el $29.9 \%$ por no tener vivienda propia.

\subsection{Talento humano}

En la consolidación de un equipo de trabajo, como lo es el conductor del vehículo compactador y su tripulación, deben intervenir factores como lo son la identificación de todos los miembros con el logro de un objetivo en común, aceptación de la importancia del rol que cada uno cumple, la buena comunicación, colaboración y un componente de afectividad que los agrupe $y$ mantenga unidos a pesar de las dificultades (Agilar; Vargas, 2010).Esta categoría permiteconocer el conjunto de valores que representan cada uno de los trabajadores, así como los institucionales, y la complementariedad o armonía entre estos; también

permite conocer aspectos relacionados con la experticia laboral y profesional del trabajador.

En términos generales se puede colegir que existe un ambiente de trabajo adecuado entre los conductores encuestados; en los resultados obtenidos, se infiere que existeafinidad $y$ cooperación entre sus compañeros, amabilidad y respeto; actitudes que propician en gran medida el buen desempeño durante el trabajo y que de ser aprovechadas y potencializadas por la empresa, pueden contribuir al mejoramiento de todos sus procesos. Sin embargo, se presenta la necesidad dentro de los mismos conductores de fortalecer sus habilidades de liderazgo para un mejor desempeño del equipo de trabajo; de esta forma se contribuiría a mejorar la seguridad de ellos y aumentaría satisfacción que sienten con su trabajo.

Un buen ambiente de trabajo, posibilita que la comunicación entre superiores y empleados se dé de manera asertiva para un buen suceso de la gestión administrativa; en este caso, los conductores consideran que pueden hablar libremente con su jefe, siempre o algunas veces, cuando están en desacuerdo con las decisiones que toma; sin embargo, un gran porcentaje de los mismos (53\%) considera que no se les brinda la posibilidad de participar en la solución de los problemas laborales que se presentan en la empresa, y además, que las ideas y sugerencias que en algún momento ofrecen, son poco o nada tenidas en cuenta (79\%).

No es suficiente entonces con escuchar las preguntas, sugerencias y reclamos de los empleados, es necesario un liderazgo proactivo de sus directivos para tratar de darle solución a sus problemas y necesidades, sean estos de cualquier índole, a través de la gestión del talento humano o del programa de bienestar laboral que toda empresa debe tener establecido. La confianza que se genera del empleado hacia su empresa cuando ve interés y atención de esta hacia sus necesidades, eleva la satisfacción frente al trabajo; además incrementa la imagen de la empresa como un gran espacio para el desarrollo de la actividad laboral. 


\section{DIAGNÓSTICO DEL CLIMA LABORAL Y ORGANIZACIONAL DE LOS CONDUCTORES DE LOS CAMPACTADORES DE LA EMPRESA DE ASEO DE MEDELLÍN- EMVARIAS}

Mario Javier Naranjo Otalvaro.

Precisamente, en referencia a la valoración que hacen de su trabajo, el $71 \%$ de los conductores considera que se sienten valorados siempre o algunas veces, por lo que es necesario alcanzar unos niveles más altos en este aspecto, ya que es un componente fundamental de la satisfacción laboral y el buen desempeño de los empleados en sus funciones. Una forma para que se sientan más valorados en la empresa, es el estímulo y reconocimiento cuando el desempeño de su labor es destacable, pues esto incentiva a un mayor esfuerzo por parte de los trabajadores e incrementa el sentido de pertenencia y compromiso hacia la empresa; pero en este aspecto hay una gran deficiencia reflejada en los resultados de la encuesta, ya que el $32 \%$ de los conductores considera que los esfuerzos extraordinarios realizados durante su trabajo nunca son reconocidos por parte de sus superiores.

Otra forma de lograr que se sientan valorados, es propiciando la inclusión de sus familias y necesidades personales del trabajador en las actividades misionales de la empresa, pues un gran porcentaje, representado por el $60.3 \%$ de los encuestados considera que su empresa nunca se preocupa por sus aspiraciones personales y familiares. Cabe anotar que un programa de bienestar laboral y de compensaciones, debe considerar el entorno inmediato del empleado como una vía efectiva para mantener un estado emocional estable tanto en su empresa, como en su núcleo familiar y social.

Un aspecto clave en la gestión del talento humano, es la evaluación objetiva del desempeño de los trabajadores, pues permite mantener los objetivos de desempeño actualizados acerca de las metas que ésta debe alcanzar; en este aspecto estudio mostró que el $50.5 \%$ de los conductores son evaluados de manera periódica, y de estos, el $21 \%$ considera que su trabajo nunca es evaluado de forma justa; mientras otro $21 \%$ considera que si lo es. Aunque el resultado anterior es similar, es importante que se preste atención a las inconformidades de los empleados, ya que estos sentimientos derivan en actitudes y acciones que pueden ir en detrimento del ambiente laboral y de las buenas prácticas laborales en la empresa. La evaluación del desempeño no puede ser vista de forma negativa

por parte del trabajador, sino que debe ser usada de manera positiva por parte del empleador para que se convierta en una buena herramienta que permita conocer las características de los empleados, sus fortalezas y debilidades y hacer planes a partir de esto, que mejoren la integración de ellos a su trabajo (Bueno, 2013).

\subsection{Organización}

Esta categoría permite evaluar las políticas institucionales relacionadas con la planeación de procesos y procedimientos implementados en la organización y que afecten directa o indirectamente las funciones desempeñadas por los conductores; se complementa, además, por la evaluación del proceso comunicativo de dichas políticas.

Frente a los procesos organizativos en la empresa, los conductores consideran que, aunque es común que se presenten cambios repentinos en su trabajo, tienen la capacidad suficiente y necesaria para adaptarse fácil y rápidamente a ellos (79\%). Este resultado indica la habilidad adaptativa que tienen la mayoría de éstos, seguramente por la amplia experiencia en el sector que les ha permitido solucionar problemas prácticos que se presentan en la rutina de trabajo. Aunque esta capacidad tiene relación directa con la edad, siendo en los jóvenes en quienes más se encuentra, el hecho de que la mayoría de conductores considere que se adapta fácilmente a los cambios, evidencia que igualmente pueden tener la capacidad de cambiar sus propios comportamientos para alcanzar ciertos objetivos y aceptar las transformaciones que proponga la empresa.

Para propiciar un buen clima organizacional, la comunicación entre los superiores y los empleados debe ser oportuna y efectiva; lo anterior implica involucrar a la alta gerencia en este proceso, ya que todos los niveles de organización en la empresa deben estar comunicados y procurar cada uno a su vez, hacer su parte para mejorar cada día más este aspecto. El balance de los resultados obtenidos para este caso, es positivo ya que la mayoría de los encuestados considera que las decisiones importantes para el buen desempeño de 
su labor, se las comunican la mayoría de las veces de forma oportuna.

La comunicación interna en las empresas debe estar dirigida no solo a informar las noticias, cambios y demandas propias del trabajo entre todos los empleados, sino que debe ser la amalgama que una todos los aspectos de la organización y dirección de la misma; y en gran medida que sirva como herramienta para mejorar el bienestar de los empleados; la comunicación debe entre otras cosas, "procurar la participación y medir el grado de satisfacción de los empleados en forma constante" (Rosenthal, s.f.).

\subsection{Valores y orgullo}

Evalúa si la misión, visión y valores de la empresa se encuentran presentes en el día a día del trabajador; también permite medir el sentido de pertenencia de los trabajadores respecto a la empresa, lo mismo el cuidado con las herramientas de trabajo. Un elemento clave generador de sentido de pertenencia por la empresa a la cual se prestan los servicios, es el conocimiento de la misión, visión y valores institucionales; la aprehensión de los mismos por parte de todos los trabajadores, es factor diferenciador para cualquier empresa, por la sencilla razón de que el trabajador es el que le da sentido real a la misma; al preguntárseles por el conocimiento de estos tres aspectos, el $60.3 \%$ manifestó conocerlos; se esperaba que fuera la totalidad de los trabajadores los que debieran conocer cuál es la misión, visión y valores que representan la empresa a la cual le están prestando sus servicios, por ser este el primer indicador de un buen clima organizacional.

Los distintos operadores responsables de hacer cumplir la misión de Emvarias, la misma que se fundamenta enla integración de los procesos de recolección, transporte, valoración, tratamiento y disposición final de residuos sólidos urbanos, aportando de manera importante al cuidado del medio ambiente, deben procurar que el impacto que dicha actividad genera sobre el mismo, sea una de las concientizaciones más altas que tengan los encargados de esta tarea fundamental para cualquier comunidad; en ese orden de ideas, es necesario destacar que el $88.6 \%$ de los conductores considera muy positivo el impacto que esta le genera al medio ambiente de la ciudad; lo cual Fiermite desarrollar entre los usuarios, aspectos claves como la cultura de la separación en la fuente; que lo dispuesto para ser recogido por el carro compactador, sea lo que realmente debe ser; de la rigurosidad en este proceso, dependerá que la cultura y el respeto por el medio ambiente, sea cada vez más aprehendida por parte de todos los ciudadanos. El porcentaje de valoración que hace la comunidad por el trabajo que realizan el equipo del carro compactador (58.2\%), puede entenderse como un indicador de que esta cultura se viene consolidando por parte de los usuarios cada vez con más éxito.

Una de las consecuencias directas de la aprehensión de la misión, visión y valores corporativos por parte de los trabajadores, es el sentido de pertenencia por la misma, que hace que se respete y valore todo lo que esta significa; para el caso en concreto, el carro compactador como principal herramienta de trabajo, es valorado significativamente por el $92.9 \%$ de los conductores; otras razones con porcentajes igualmente altos fueron: el cuidar el carro porque disfrutan lo que hacen; por mantenerlo en buenas condiciones mecánicas; porque son muy costosos; porque significa una apropiación y disfrute por lo que hacen y por la empresa. Adicional a ello manifiestan conocer y cumplir las condiciones de operación segura del vehículo en un porcentaje importante (98.4\%) y que reportan a tiempo las anormalidades mecánicas que presenta el vehículo en un porcentaje similar (97.3\%); el tener este cuidado por su principal herramienta de trabajo, indica un gran respeto por la empresa, por lo que hacen y por la comunidad, algo digno de ser valorado y reconocido por parte de sus superiores.

\subsection{Capacitación}

Con esta dimensión se pretenden conocer las aspiraciones de formación y cualificación personal y profesional de los conductores, que contribuyan a consolidar su proyecto de vida, el empoderamiento institucional, la eficiencia y eficacia en el desarrollo de las funciones propias de Emvarias.

La capacitación es una dimensión estratégica que permite identificar y ejecutar acciones que atiendan las necesidades de 


\section{DIAGNÓSTICO DEL CLIMA LABORAL Y ORGANIZACIONAL DE LOS CONDUCTORES DE LOS CAMPACTADORES DE LA EMPRESA DE ASEO DE MEDELLÍN- EMVARIAS}

Mario Javier Naranjo Otalvaro.

entrenamiento, formación y desarrollo de las personas que integran cada uno de los equipos de trabajo; se pueden mencionar las siguientes acciones: actualizar en temas tecnológicos, de Ley y funcionales; ofrecer espacios de creación e innovación que aporten al mejoramiento de la gestión; promover eventos orientados al mejoramiento de las competencias de cada uno de los funcionarios de la empresa; diseñar programas ajustados al entrenamiento de competencias laborales específicas de acuerdo a las funciones y responsabilidades (Vecino, 2012).

Las empresas del sector público están obligadas por disposiciones legales (Ley 909 de 2004) a contemplar planes de capacitación permanente a todos sus trabajadores, porque el conocimiento en la actual sociedad de la información y del conocimiento, está evolucionando cada vez con mayor rapidez y la obsolescencia del mismo está a la orden del día, si no se tiene la cultura de la información y de la formación oportuna, las oportunidades se van perdiendo porque las nuevas generaciones, más formadas, están irrumpiendo cada vez con más fuerza en el mercado laboral. Frente a esta pregunta, el $94 \%$ de los conductores considera necesario capacitarse para potenciar su desarrollo personal y proyecto de vida; un porcentaje similar lo considera necesario capacitarse en algún área de su interés para poder mejorar su desempeño laboral, en temas relacionados con la mecánica diésel para la mayoría de los encuestados (76.1\%); mecánica automotriz (66.3\%); eléctrica (41.8\%); humanidades (30.4\%); informática (26.1\%); logística (25.5\%); electrónica (17.4\%); mecánica industrial (15.8\%); soldadura (15.8\%), entre otros.

Dicha capacitación aspiran adelantarla a través de cursos cortos de seis meses (40\%); técnicas de dos años (23.9\%); ingenierías o carreras profesionales (19.4\%) y tecnologías de tres años (16.7\%).

\subsection{Servicio al cliente}

Permite evaluar el nivel de correspondencia del trabajador con la comunidad a la que presta sus servicios, con base en la relación de reciprocidad que debe haber entre ambos actores; busca satisfacer e incluso sobrepasar las expectativas y necesidades del mismo; comprende aquellas actividades que facilitan al cliente estar en contacto con las áreas de la empresa, de las cuales requiere soluciones y respuestas (Evia, 2011).

La satisfacción plena del cliente es la razón fundamental de toda empresa, y mucho más una empresa que tiene como misión la recolección, transporte, valoración, tratamiento y disposición final de residuos sólidos urbanos; misión desarrollada a través de los operadores o contratistas que tienen asignada tarea tan importante; en ese orden de ideas, los conductores consideran que la relación que tienen con la comunidad es buena (97.3\%), factor clave para lograr profundizar el amor que se siente por habitar una ciudad como Medellín, que se precia de tener uno de los mejores sistemas a nivel nacional de recolección y disposición final de sus residuos sólidos; lo que permite un mayor empoderamiento de la ciudadanía aumentando más su sentido de pertenencia no sólo por la empresa, así sea a través de terceros, sino por la ciudad toda; como resultado de lo anterior, un porcentaje similar (83.7\%) manifiesta no haber tenido ningún tipo de problemas con la comunidad a la cual atienden; refleja lo anterior que la dimensión del servicio al cliente, se desarrolla con niveles altos de aprobación por parte de la comunidad.

Es bien sabido que los clientes no siempre están satisfechos con lo que se les brinda; a pesar de que el grado de satisfacción es muy positivo; es necesario señalar que un $15.2 \%$ manifiesta haber tenido algún tipo de dificultad con la comunidad por la prestación del servicio de aseo; los más significativos señalados por los conductores son:

- Retrasos en los horarios de recolección

- Fugas de lixiviados del vehículo

- Los carros recolectores estorban en las vías públicas

- La gente quiere que les recojan artículos que no se deben como colchones y escombros

- Sacan la basura a destiempo.

Este tipo dificultades deben ser atendidas con urgencia por parte de la alta dirección de los distintos operadores del servicio de aseo en la ciudad, para ser corregidas de manera oportuna, porque un problema que no se resuelve a tiempo, va creciendo en magnitud y complejidad y su 
solución se hace más difícil y costosa; los responsables del aseo en la ciudad deben ser conscientes que Medellín es la ciudad del país con el mejor sistema de recolección de sus residuos; y problemas como los manifestados por los conductores, empiezan a generar malestar en la comunidad, ya que el servicio de aseo público es clave para la calidad de vida de cualquier comunidad.

\subsection{Preguntas valorativas}

Son preguntas de control que sirven para contrastar algunos de los resultados obtenidos en las preguntas planteadas en las distintas dimensiones contempladas en la encuesta; definiéndose cuatro temas claves con una valoración en una escala de 1 a 10 , donde 1 es muy malo y 10 es excelente; siendo éstos: los vehículos, los superiores, sus compañeros y la satisfacción con su empleo.

El dato más significativo resulta de la valoración que hacen de los vehículos en la escala entre 6 y 10, representa numéricamente una valoración positiva del 54.6\%; contrasta este resultado con la pregunta que se les hizo en la dimensión relativa a los valores y orgullo sobre el cuidado que le dan al vehículo que conducen, la que fue respondida con un $92.9 \%$ en términos de ser muy valorado porque es su principal herramienta de trabajo; es claro que ambos resultados no guardan una relación proporcional, aspecto que debe llamar la atención de los superiores inmediatos de los conductores, para que revisen este punto en particular con los encargados de la supervisión técnica de los vehículos, pues es factible que dada la incongruencia en una pregunta que debiera dar una respuesta parecida, los vehículos no estén siendo cuidados como debe ser por parte de quien los opera.

\section{Conclusiones:}

Lo que el análisis de los resultados obtenidos a nivel global revela, es que no se puede trabajar por mejorar una dimensión o aspecto en particular en el funcionamiento de la empresa, sin intervenir también en las demás dimensiones analizadas. Así la comunicación interna, el trabajo en equipo, los programas de bienestar laboral, las evaluaciones de desempeño, entre otras características no menos importantes, son componentes del funcionamiento de una empresa que están relacionados y se influyen mutuamente. El mejoramiento en cualquier aspecto humano de la organización empresarial, debe ir acompañado por una evaluación y valoración de los demás componentes, para así asegurar que los cambios que se den dentro de la empresa a favor del bienestar de los empleados, sean realmente productivos y duraderos.

Es muy importante que Emvarias considere la posibilidad de formalizar un futuro contrato de operación del servicio de aseo y disposición final de los residuos sólidos en la ciudad de Medellín, con un solo operador, para que las condiciones laborales y el ambiente de trabajo de los conductores sean iguales para todos; lo anterior permitiría una mayor optimización del talento humano que se reflejaría en un mejor ambiente laboral y organizacional, aumentaría el sentido de pertenencia hacia la empresa, se mejoraría la productividad, y la ciudad sería la gran beneficiada.

\section{Referencias:}

Aguilar, J; Vargas, J(2010). Trabajo en equipo. Network de psicología organizacional. Tomado de: http://www.conductitlan.net/psicolo gia_organizacional/trabajo_en_equi po.pdf el día 19 de mayo del 2014.

Bueno, M (s.f.). Evaluación de desempeño como visualizador de rendimiento. Tomado de: http://www.eoi.es/blogs/mintecon/ 2013/06/03/evaluacion-dedesempeno-como-visualizador-derendimiento/ el día 19 de mayo del 2014.

Evia, I (2011). Influencia del Clima Organizacional en el Servicio al Cliente.Tomado de: http://jupiter.utm.mx/ tesis_dig/11 492.pdf el día 26 de mayo de 2014. 
Factores de riesgo para el consumo de tabaco en una población de adolescentes escolarizados. Rubines Juárez Ángel Orlando. Tesis UNMSM.Tomado de:http://sisbib.unmsm.edu.pe/bibvi rtualdata/tesis/salud/Rabines_J_A/C AP\%C3\%8DTULO1-

introduccion.pdf. El día 21 de mayo de 2014.

García, D (2011). Estudio de la motivación y satisfacción laboral en el colectivo de operadores de grúa torre en edificación a través de un método cualitativo taller de dirección y gestión de recursos humanos en la edificación. Tomado de: http://riunet.upv.es/bitstream/handl e/10251/13547/GARCIA\%20MENEN DEZ,\%20DEBORAH.pdf?sequence $=2$ el día 23 de mayo de 2014.

Ley 909 de 2004. Por la cual se expiden normas que regulan el empleo público, la carrera administrativa, gerencia pública y se dictan otras disposiciones. Colombia.

Rosenthal, V. (S.f.). La comunicación interna: gestión de vital importancia dentro de las organizaciones. Tomado de: http://www.ideared.org/images/Art $\% 20$ Int $\% 203 \% 20$ Comunicacion $\% 20 i$ nterna.pdf el día 19 de mayo del 2014

Vecino, M (2012). Dimensión estratégica de la capacitación.Tomado de: https://jcvalda.wordpress.com/2012 /10/01/dimension-estrategica-de-lacapacitacion el día 26 de mayo de 2014. 
Face

Volumen $15 N^{\circ}$ 1, Año 2015 